\title{
Combining adverse pregnancy and perinatal outcomes for women exposed to antiepileptic drugs during pregnancy, using a latent trait model
}

Xuerong Wen ${ }^{1 *} \mathbb{D}$, Abraham Hartzema ${ }^{2}$, Joseph A. Delaney ${ }^{3}$, Babette Brumback ${ }^{4}$, Xuefeng Liư ${ }^{5}$, Robert Egerman ${ }^{6}$, Jeffrey Roth', Rich Segal ${ }^{2}$ and Kimford J. Meador ${ }^{8}$

\begin{abstract}
Background: Application of latent variable models in medical research are becoming increasingly popular. A latent trait model is developed to combine rare birth defect outcomes in an index of infant morbidity.

Methods: This study employed four statewide, retrospective 10-year data sources (1999 to 2009). The study cohort consisted of all female Florida Medicaid enrollees who delivered a live singleton infant during study period. Drug exposure was defined as any exposure to Antiepileptic drugs (AEDs) during pregnancy. Mothers with no AED exposure served as the AED unexposed group for comparison. Four adverse outcomes, birth defect (BD), abnormal condition of new born (ACNB), low birth weight (LBW), and pregnancy and obstetrical complication (PCOC), were examined and combined using a latent trait model to generate an overall severity index. Unidimentionality, local independence, internal homogeneity, and construct validity were evaluated for the combined outcome.

Results: The study cohort consisted of 3183 mother-infant pairs in total AED group, 226 in the valproate only subgroup, and 43,956 in the AED unexposed group. Compared to AED unexposed group, the rate of BD was higher in both the total AED group (12.8\% vs. $10.5 \%, P<.0001)$, and the valproate only subgroup (19.6\% vs. 10. $5 \%, P<.0001)$. The combined outcome was significantly correlated with the length of hospital stay during delivery in both the total AED group (Rho $=0.24, P<.0001$ ) and the valproate only subgroup (Rho $=0.16, P=.01$ ). The mean score for the combined outcome in the total AED group was significantly higher ( $2.04 \pm 0.02 \mathrm{vs} .1 .88 \pm 0.01$, $P<.0001)$ than AED unexposed group, whereas the valproate only subgroup was not.

Conclusions: Latent trait modeling can be an effective tool for combining adverse pregnancy and perinatal outcomes to assess prenatal exposure to AED, but evaluation of the selected components is essential to ensure the validity of the combined outcome.
\end{abstract}

Keywords: Latent trait model, Antiepileptic drugs, Valproate, Adverse pregnancy outcome, Adverse perinatal outcome, Combining outcomes

\footnotetext{
* Correspondence: xuerongwen@uri.edu

${ }^{1}$ Health Outcomes, College of Pharmacy, University of Rhode Island, 7

Greenhouse Rd., Kingston, RI 02881, USA

Full list of author information is available at the end of the article
}

(c) The Author(s). 2017 Open Access This article is distributed under the terms of the Creative Commons Attribution 4.0 International License (http://creativecommons.org/licenses/by/4.0/), which permits unrestricted use, distribution, and reproduction in any medium, provided you give appropriate credit to the original author(s) and the source, provide a link to the Creative Commons license, and indicate if changes were made. The Creative Commons Public Domain Dedication waiver (http://creativecommons.org/publicdomain/zero/1.0/) applies to the data made available in this article, unless otherwise stated. 


\section{Keypoints}

- AEDs have significant effects on all four component birth outcomes, and as well as the combined outcome.

- Valproate has significant effects on two out of four component outcomes, and no association with the combined outcome.

- Latent Trait Modeling is an effective tool to combine rare birth defect outcomes.

- Evaluation of selected components is essential to ensure the validity of the combined outcome.

\section{Background}

Birth defects (BDs), involving major congenital malformation (MCM) and minor anomaly (MA) are the leading causes of infant mortality, morbidity, and years of potential life lost. In the USA, the association of infant BDs and pregnancy and obstetrical complications (PCOCs) with maternal exposure to antiepileptic drugs (AEDs) has been investigated extensively [1-3]. However, the rare occurrence of BDs, abnormal condition of new born (ACNBs), and PCOCs limits the power of most published studies, and makes study results inconclusive [4-6]. A joint model for combining individual outcomes is proposed to improve the efficiency and power of BD studies [7].

Latent variable models have increasingly been applied in medical research, including measurement of quality of life, diagnostic testing, survival analysis, and joint modeling of longitudinal data [8]. Latent variables are unobserved variables that can only be assessed indirectly by observable manifest variables. A latent variable model is a statistical approach that uses a set of observable manifest variables to derive one or more unobsersable variables. In latent variable model with a latent trait setting, the manifest variables are discrete, including dichotomous, nominal, or ordinal variables, whereas, the latent variables are continuous variables and can be assumed as normally or log-normally distributed [9]. An important assumption for latent variable model is the "local independence", defined as that the manifest variables are conditionally independent upon a given latent variable, and the relationship among the manifest variables is fully explained by the latent variable [10]. A latent variable model in a latent trait setting was developed for this study to combine individual BD outcomes and generate an infant morbidity index [11]. This model combines four infant morbidity outcomes and generates a continuous index representing the infant's propensity for morbidity [11]. Application of this model to combine rare adverse pregnancy and perinatal outcomes in drug safety studies may increase statistical power and improve efficiency of studies investigating low prevalence sequelae.
A debate remains over the use of combined or individual outcomes in drug safety studies. A combined outcome may lead to incorrect results and threaten the validity of the study if the components are selected inappropriately $[12,13]$. Therefore, the combined outcome must be evaluated in terms of conceptualization of the composite outcome [12], and appropriate properties of the latent variable, such as local independence, construct validity and reliability [14].

The objective of this study is to apply a latent trait model to generate a valid combined outcome (adverse perinatal and pregnancy outcome; APO) to assess the overall adverse pregnancy and perinatal risks for mothers and infants exposed to AEDs.

\section{Methods}

Data sources

This study used four statewide, retrospective 10-year databases: Florida Medicaid claims, Florida Birth Vital Statistics, Florida Birth Anomalies, and Florida Hospital Discharge Inpatient and Outpatient records (January 1, 1999-December 31, 2009).

\section{Study population}

This study includes all female Florida Medicaid enrollees who delivered a live singleton infant between April 1, 2000 and December 31, 2009. Exclusion criteria for maternal-infant pairs are: mothers with dual eligibility for Medicare, HMO, or private insurance; mothers having multiple births (twins or higher order); mothers with diabetes mellitus (ICD-9 codes: 249.x, 250.x, 790.29, or use of any anti-diabetics during baseline), hypertension (ICD-9 codes: 401.x, 416.x, 796.2, 997.91, 459.3, or antihypertensive drug use during baseline), or HIV prepregnancy (ICD-9 codes: 042, 079.53, V08, V01.79, 795.71, or use of any antiretroviral therapy); infants who were twins, triplets, quadruplets or more; infants with birth weight lower than $350 \mathrm{~g}$ or higher than $6000 \mathrm{~g}$; mothers or infants with critical information missing (e.g., birth weight, demographics, or medical information).

\section{Study design}

The index date is the infant's birth date. The drug exposure window was defined as the preceding 9-month pregnancy period after the first day of the last menstrual date. A six month baseline period before the first date of the last menstrual date was utilized to determine the baseline demographic and clinical characteristics. BD outcomes were detected 0-365 days after live birth.

\section{Exposure}

Drug exposure was determined from Medicaid pharmacy claims using national drug codes. Two drug 
exposure groups, valproate and AEDs (including valproate), were employed to develop two scenarios with different patterns of association with the four component outcomes. Valproate use was defined as prescriptions dispensed for valproate, sodium valproate, or divalproex. AEDs included: carbamazepine, ethosuximide, felbamate, gabapentin, lamotrigine, levetiracetam, oxcarbazepine, phenobarbital, phenytoin, pregabalin, primidone, tiagabine, topiramate, valproate, and zonisamide.

The birth anomalies are related to exposure time during pregnancy: [15] MCM associates with teratogen exposure in the first trimester [16], and MA and LBW relate to the maternal drug exposure in the third trimester [15, 17]. Therefore, maternal drug exposure during the entire pregnancy can affect the combined outcome. The prenatal drug exposure window was established as the period of 14 days before the first day of the mother's last menstrual period to the infant's birth date. The drug exposure was defined as any one dose of the drugs listed above dispensed during the exposure window, including which drug was dispensed prior to the exposure window and its days of supply covers at least one day of the exposure window. Adding 14 days prior to the pregnancy takes into account the conception period and the residual effects of AEDs. Sensitivity analysis was conducted to examine the effects of different drug exposure windows on the combined outcome.

\section{Component outcomes}

We investigated four adverse pregnancy and infant outcomes: BD (involving MCM and MA), abnormal condition of new born (ACNB), LBW, and PCOC from multiple data sources. The operational definition for each component outcome was listed in Additional file 1: Table S1. MCMs and MAs were collected for 365 days following birth using the $9^{\text {th }}$ edition of the International Classification of Diseases-Clinical Modification (ICD-9 CM) code (740-759.9) from Florida Hospital Discharge Inpatient and Outpatient data. It has been confirmed that Hospital Discharge data, along with other Children's Medical Services diagnostic information, efficiently enhanced case ascertainment for BD cases from Florida Birth Vital Statistics data [18-20]. ACNB and birth weight were obtained from Florida Birth Vital Statistics. The common conditions of ACNBs include anemia, birth injury, fetal alcohol syndrome, hyaline membrane disease, and assisted ventilation. Birth weight was categorized into four levels: Extremely Low Birth Weight (ELBW, 350-999 g), Very Low Birth Weight (VLBW, 1000-1499 g), Low Birth Weight (LBW, 1500-2499 g) and Normal Birth Weight (NBW, 2500-5999 g). PCOCs were identified either from Florida Birth Vital Statistics data or using ICD-9-CM and Current Procedural
Terminology codes from Medicaid inpatient and outpatient claims data depending upon the extent of the validity and reliability of these data sources as reported in previous studies [21-25]. Gestational hypertension, preeclampsia, and eclampsia were identified using ICD9-CM codes from hospital discharge data [22, 23]. Preterm birth was operationally defined as gestational age less than 37 weeks [24]. Gestational age was computed from the infant birth date and mother's last menstrual period. To identify obstetrical conditions, we defined cesarean delivery and forceps or vacuum extractor delivery from either birth certificates or ICD-9-CM codes in hospital discharge data, if it was missing in the birth certificates. Postpartum hemorrhage was identified solely using ICD-9-CM codes in hospital discharge data due to poor validity of birth certificate data on pregnancy complications and obstetric events [25].

Selected component outcomes were evaluated for similarity of importance, frequency rate, and treatment effect. The importance of the component outcome was assessed by computing Spearman correlations between individual outcomes and a clinically meaningful endpoint, defined as infant's length of hospital stay following delivery [26].

\section{Reference group and covariates}

A reference group, defined as infants with no maternal exposure to any AEDs during pregnancy and termed "AED unexposed group", was selected for the estimation of treatment effects of the combined and component outcomes. The potential confounding factors were controlled using propensity score matching techniques. Previous studies have documented that common risk factors for adverse maternal and infant outcomes include socioeconomic status, infant gender, maternal age, race, BMI, smoking, alcohol consumption, parity, and drug exposure during pregnancy [27-30]. Significant teratogens such as alcohol and tobacco were controlled for during treatment effect assessment [31-36]. Other medical indications documented as teratogens in previous studies were also controlled in this study [37, 38]. Demographic characteristics were identified from birth certificates, whereas co-morbidities or co-medications during pregnancy were identified using ICD-9-CM and National Drug Codes from Hospital Discharge data.

\section{Combining outcomes using latent trait modeling}

The statistical inference and mathematical algorithm for the model have been described elsewhere [39]. An important assumption of the model is "local independence", defined as an independence of manifest outcomes conditioned on latent variables [11]. Estimated Generalized Nonlinear Least Squares estimation was employed to obtain the parameters involved in the 
latent trait model $[11,40]$. The derivative process for the combined outcome is as follows:

Step 1. Calculate initial estimates of the model parameters. First, we selected initial estimates to make the iteration process converge. We obtained 32 independent levels by combining 3 dichotomous component outcomes: BD (Yes/No), ACNB (Yes/No), PCOC (Yes/No), and 1 polytomous component outcome: Birth Weight (BW): 2500 5999 g, 1500 2499 g, $1000 \sim 1499$ g, $350 \sim 999$ g. The frequencies and proportions for each level of the combination of four component outcomes were calculated and utilized to deduce the initial estimates of the model parameters. Step 2. Derive the final estimates of the model parameters. Using the set of initial values and the modified Gauss-Newton algorithm, final estimates of the model parameters were obtained. The modified Gauss-Newton algorithm was run in SAS Proc IML, starting from the initialized value at iteration 0 , until the difference of the last two estimates was less than $10^{-9}$. All final parameters were estimated from the iteration process.

Step 3: Calculate the conditional probabilities given the latent variable $\mathrm{S}$ for each component outcome. Substituting the final estimates into the latent trait model, we calculated expected probabilities and counts for each level of the combination of four component outcomes.

Step 4: Derive the combined outcome, the severity index of adverse perinatal and pregnancy outcome (APO). Substituting final estimates and conditional probabilities into the latent trait model, we further obtained the posterior distribution of latent variable $S$, and the mean of the posterior distribution $(\hat{s})$. The final estimate, APO, is a rescaled $\hat{s}$, to adapt for measurement of severity of health status.

\section{Evaluation of combined outcome}

Local independence of four component outcomes was assessed using Yen's Q statistics [41]. Validity and reliability of the combined outcome were evaluated using factor analysis and Spearman correlation [42, 43].

\section{Statistical analysis}

Continuous variables were compared using a student $t$ test, and categorical variables were examined using a chi-square test. Spearman correlation was calculated for discrete data, and Pearson correlation was calculated for continuous variables that are normally distributed. Multivariate logistic modeling was used to obtain propensity scores and assess the effects of drug use for each component outcome. Latent trait modeling was employed to combine four component outcomes into a severity index.

Statistical analysis was conducted using SAS 9.3 (Cary, NC). $P<0.05$ was considered a statistically significant difference, except where $P<0.025$ was deemed significant after Bonferroni correction for two comparisons.

\section{Results}

After applying all inclusion and exclusion criteria, the final study cohort consisted of 3183 mother-infant pairs in the AED exposure group, 226 mother-infant pairs in the valproate exposure subgroup, and 43,956 motherinfant pairs in the AED unexposed group. A comparison of the demographic and clinical characteristics of the three groups is presented in Table 1 , and the characteristics of all study populations, as well as missing data, were presented in Additional file 1: Table S2. The detailed data about AED exposure in pregnant women in Florida Medicaid has been published in elsewhere [44].

The combined outcome, APO scores were compared between AED, valproate only, and AED unexposed group (Fig. 1). The average APO score in the total AED group was significantly different for AED unexposed group (Mean \pm SE: $2.04 \pm 0.02$ vs $1.88 \pm 0.01, P<.0001$ ), but not for the valproate subgroup (Mean \pm SE: $2.00 \pm$ 0.07 vs. $1.88 \pm 0.01, P=0.1003)$. The valproate subgroup $(n=226)$ was smaller than the total AED group $(n=3183)$, which could have affected the statistical results due to insufficient power.

Figure 2 presents the incidence rates of PCOC, BD (MCM and MA), and ACNB in three study groups. Compared to AED unexposed group, the total AED exposed group had significant higher rates on PCOC (36\% vs. $28 \%$, $P<.0001)$ and ACNB $(12.1 \%$ vs. $7.8 \%, P<.0001)$. The rate of $\mathrm{PCOC}$ was not significantly higher in the valproate subgroup compared to the AED unexposed group ( $34 \%$ vs. $28 \%, P=0.0509)$. The valproate subgroup had the highest rates of $\mathrm{BD}$, significantly higher than the AED unexposed group (20\% vs. $10.5 \%, P<.0001)$. ACNB in valproate subgroup was not different than the AED unexposed group (10.2\% vs. $7.8 \%, P=0.1525)$.

Figure 3 delineates the distribution of four BW categories (Normal: 2500-5999 g, LBW: $1500 \sim 2500$ g, VLBW: $1000 \sim 1500 \mathrm{~g}$, and ELBW: $<1000 \mathrm{~g})$ in three study groups. The rate of LBW in the total AED exposed group was significantly higher than that of the AED unexposed group (88.1\% vs. $91.6 \%, 10.6 \%$ vs. $6.7 \%$, $0.9 \%$ vs. $0.7 \%, 0.5 \%$ vs. $0.99 \%, P<.0001)$. The valproate subgroup did not differ significantly in the distribution of BW categories from AED unexposed group $(88.6 \%$ vs. $91.6 \%, 10.6 \%$ vs. $6.7 \%, 0.4 \%$ vs. $0.7 \%, 0.4 \%$ vs. $0.99 \%$, $P=0.0752)$. 
Table 1 Demographic and Clinical Characteristics of Study Participants. Obtained from Florida Birth Vital Statistics or Medicaid Claims Data

\begin{tabular}{|c|c|c|c|c|c|}
\hline Characteristics & $\begin{array}{l}\text { Valproate } \\
\text { Sub-group } \\
N=226\end{array}$ & $\begin{array}{l}\text { Total AED } \\
\text { Group }^{a} \\
N=3183\end{array}$ & $\begin{array}{l}\text { AED Unexposed } \\
\text { Group } \\
N=43,956\end{array}$ & $P$ Value ${ }^{* *}$ & $P$ Value*** \\
\hline Maternal age at infant born, Mean \pm SD & $25.9 \pm 6.4$ & $26.5 \pm 6.0$ & $24.6 \pm 5.2$ & $<.0001$ & 0.0014 \\
\hline Father's age at infant birth, Mean \pm SD & $52.1 \pm 33.2$ & $47.5 \pm 30.7$ & $43.3 \pm 29.7$ & $<.0001$ & $<.0001$ \\
\hline \multicolumn{6}{|l|}{ Mother's Race, N (\%) } \\
\hline White & $177(72)$ & $2200(69)$ & $20,333(46)$ & \multirow[t]{3}{*}{$<.0001$} & \multirow[t]{3}{*}{$<.0001$} \\
\hline Black & $27(11)$ & $429(13)$ & $13,991(32)$ & & \\
\hline Others & $41(17)$ & $552(17)$ & $9550(22)$ & & \\
\hline \multicolumn{6}{|l|}{ Father's Race, N (\%) } \\
\hline White & $95(39)$ & $1455(46)$ & $15,669(36)$ & \multirow[t]{3}{*}{$<.0001$} & \multirow[t]{3}{*}{$<.0001$} \\
\hline Black & $31(13)$ & $327(10)$ & $9733(22)$ & & \\
\hline Others & $32(13)$ & $474(15)$ & $8024(18)$ & & \\
\hline \multicolumn{6}{|l|}{ Father's education level, $N(\%)$} \\
\hline Above High School & $66(40)$ & $765(33)$ & $14,219(41)$ & $<.0001$ & 0.7107 \\
\hline Mother's previous adverse pregnancy experience, $N(\%)$ & $1(0.7)$ & $23(1)$ & $307(2)$ & 0.0237 & 0.3482 \\
\hline Mother's receipt of any prenatal care, $N(\%)$ & $143(99)$ & $2172(99)$ & $18,590(98)$ & 0.5571 & 0.0246 \\
\hline Mother's total number of prenatal visits, Mean \pm SD & $11.1 \pm 18.2$ & $11.3 \pm 16.9$ & $8.8 \pm 15.7$ & $<.0001$ & 0.0059 \\
\hline Mother's marital status, Yes, N (\%) & $86(35)$ & $1137(36)$ & $18,041(41)$ & $<.0001$ & 0.1679 \\
\hline Mother's parity (previous live births), Mean \pm SD & $1.1 \pm 1.3$ & $1.3 \pm 5.1$ & $1.7 \pm 3.7$ & $<.0001$ & $<.0001$ \\
\hline Mother's tobacco use, N (\%) & $74(31)$ & $936(30)$ & $7133(16)$ & $<.0001$ & $<.0001$ \\
\hline Mother's average tobacco use, Mean \pm SD & $4.8 \pm 15.9$ & $4.0 \pm 13.5$ & $1.7 \pm 9.0$ & $<.0001$ & 0.0028 \\
\hline Mother's alcohol use, $N(\%)$ & $3(1)$ & $38(1)$ & $175(0.4)$ & $<.0001$ & 0.0183 \\
\hline \multicolumn{6}{|l|}{ Mother's education level, $N(\%)$} \\
\hline Above High School & $86(37)$ & $1173(37)$ & $16,772(39)$ & 0.0980 & 0.5377 \\
\hline Infant male gender, $N(\%)$ & $120(49)$ & $1549(49)$ & $19,048(43)$ & $<.0001$ & 0.0001 \\
\hline Mother's previous gestational diabetes, N (\%) & $6(4)$ & $84(4)$ & $604(3)$ & 0.1714 & 0.4170 \\
\hline Mother's previous gestational hypertension, $N(\%)$ & $6(3)$ & $138(5)$ & $1240(3)$ & $<.0001$ & 0.2559 \\
\hline Mother's previous cesarean, N (\%) & $17(13)$ & $306(14)$ & $3056(17)$ & 0.0005 & 0.1819 \\
\hline Mother's Epilepsy diagnosis during baseline and pregnancy, $N(\%)$ & $60(24)$ & $571(18)$ & $81(0.2)$ & $<.0001$ & $<.0001$ \\
\hline Mother's Anxiety diagnosis during baseline and pregnancy, N (\%) & $15(6)$ & $230(7)$ & $218(0.5)$ & $<.0001$ & $<.0001$ \\
\hline Mother's Neural Pain diagnosis during baseline and pregnancy, N (\%) & $0(0)$ & $27(0.9)$ & $27(0.1)$ & $<.0001$ & $>.999$ \\
\hline Mother's Bipolar diagnosis during baseline and pregnancy, N (\%) & $56(23)$ & $444(14)$ & $499(1.1)$ & $<.0001$ & $<.0001$ \\
\hline Mother's Depression diagnosis during baseline and pregnancy, N (\%) & $21(9)$ & $328(10.3)$ & $743(1.7)$ & $<.0001$ & $<.0001$ \\
\hline Mother's Migraine diagnosis during baseline and pregnancy, N (\%) & $12(5)$ & $96(3)$ & $173(0.4)$ & $<.0001$ & $<.0001$ \\
\hline Mother's mental disorder diagnoses during baseline and pregnancy, N (\%) & $90(37)$ & $1118(35)$ & $3777(9)$ & $<.0001$ & $<.0001$ \\
\hline Mother's antipsychotic exposure during pregnancy, N (\%) & $50(20)$ & $338(11)$ & $275(0.6)$ & $<.0001$ & $<.0001$ \\
\hline Mother's antidepressants exposure during pregnancy, N (\%) & $90(37)$ & $886(28)$ & $1281(3)$ & $<.0001$ & $<.0001$ \\
\hline Mother's folic acid use during pregnancy, $N(\%)$ & $150(61)$ & $1965(62)$ & $17,948(41)$ & $<.0001$ & $<.0001$ \\
\hline $\begin{array}{l}\text { Mother's anxiolytics (including sedatives and hypnotics) } \\
\text { exposure during pregnancy, } N(\%)\end{array}$ & $76(31)$ & $1832(58)$ & $1452(3)$ & $<.0001$ & $<.0001$ \\
\hline $\begin{array}{l}\text { Number of hospitalization for seizure during pregnancy, } \\
\text { Median (min, max) }\end{array}$ & $0(0,4)$ & $0(0,6)$ & $0(0,3)$ & $<.0001$ & $<.0001$ \\
\hline Number of physician visits with seizure diagnoses during pregnancy, & $0(0,3)$ & $0(0,5)$ & $0(0,6)$ & $<.0001$ & 0.0208 \\
\hline
\end{tabular}


Table 1 Demographic and Clinical Characteristics of Study Participants. Obtained from Florida Birth Vital Statistics or Medicaid Claims Data (Continued)

\begin{tabular}{|c|c|c|c|c|c|}
\hline $\begin{array}{l}\text { Mother's infection and parasitic diagnosis during baseline } \\
\text { and pregnancy, } N(\%)^{b}\end{array}$ & $30(12)$ & $355(11)$ & $2959(7)$ & $<.0001$ & 0.0006 \\
\hline Mother's antibiotics exposure during pregnancy, N (\%) & $117(48)$ & $1418(45)$ & $13,854(32)$ & $<.0001$ & $<.0001$ \\
\hline
\end{tabular}

${ }^{a}$ By definition, total AED group includes the patients who used valproate

bInclude including: Virus, Rubella, Cytomegalovirus, HIV, Syphilis, Herpes simplex virus, Toxoplamosis, Varicella virus, Venezuelan equine encephalitis virus, Phenylketonuria, Hypoxia

**Compared between total AED group and AED unexposed group

***Compared between valproate subgroup and AED unexposed group

In Table 2, we further compared the propensity score adjusted drug effects and clinical importance (defined as Spearman correlation with the length of hospital stay during delivery) for each component or combined outcome in the total AED group or valproate subgroup. The adjusted drug effects and Spearman correlation with the length of hospital stay during delivery were significant for the total AED group on all four component outcomes, and for the valproate subgroup on $\mathrm{BD}$ and PCOC, but not for ACNB or LBW. The combined outcome APO was significantly associated with exposure to total AED $(\beta \pm$ SE: $0.24 \pm 0.03, P<.0001)$ or valproate only $(\beta \pm$ SE: $0.32 \pm 0.09, P=.0004)$.

Expected and observed frequencies and percentages of each combination of the four observed outcomes were enumerated in Additional file 1: Table S3. Additional file 1: Table S3 also presents the estimated posterior mean $\hat{s}$ and final estimate APO for $32 \mathrm{com}$ binations of four component outcomes, each of which is associated with an unique score of APO, ranging from 1 to 8 .
There was no correlation between the four component outcomes after controlling for the latent variable. Thus, local independence of the four component outcomes was established according to Yen's $\mathrm{Q}_{3}$ Statistics. The internal homogeneity was confirmed in all four component outcomes. They all significantly correlate with each other and the combined outcome APO. APO was significantly correlated with the length of hospital stay during delivery (Rho $=0.27, P<.0001$ ), and no correlation with infant breast fed status (Rho = $-0.07, P<.0001)$ indicate that APO was associated with a well-established health status measure. The higher the APO score, the longer the hospital stay for the mothers and infants during delivery.

\section{Sensitivity study}

We re-defined the pregnancy period calculating gestational age +10 day, 20 days, and 30 days to examine the change in association between AED exposure during pregnancy and four component outcomes. There were no significant differences between these time windows.

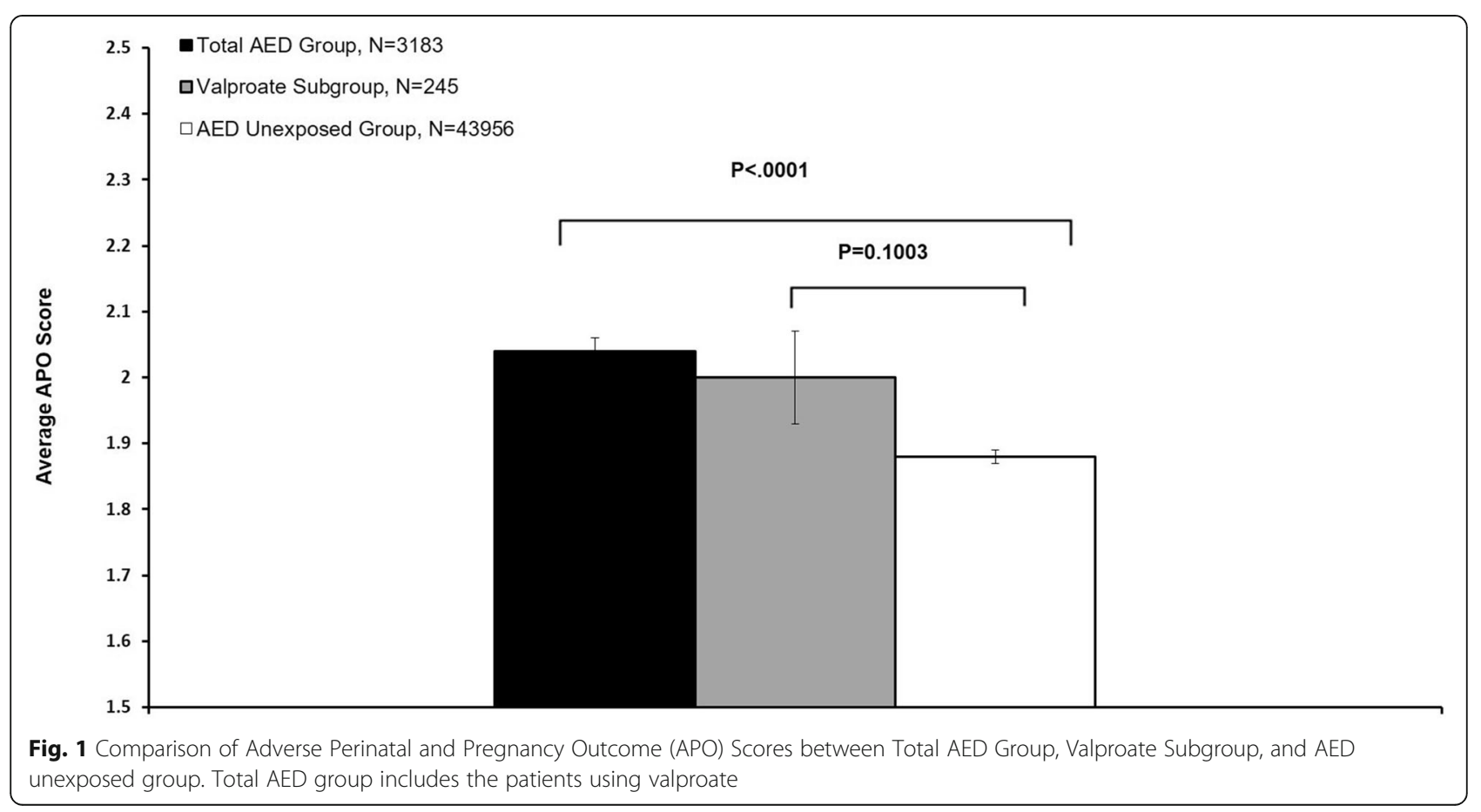




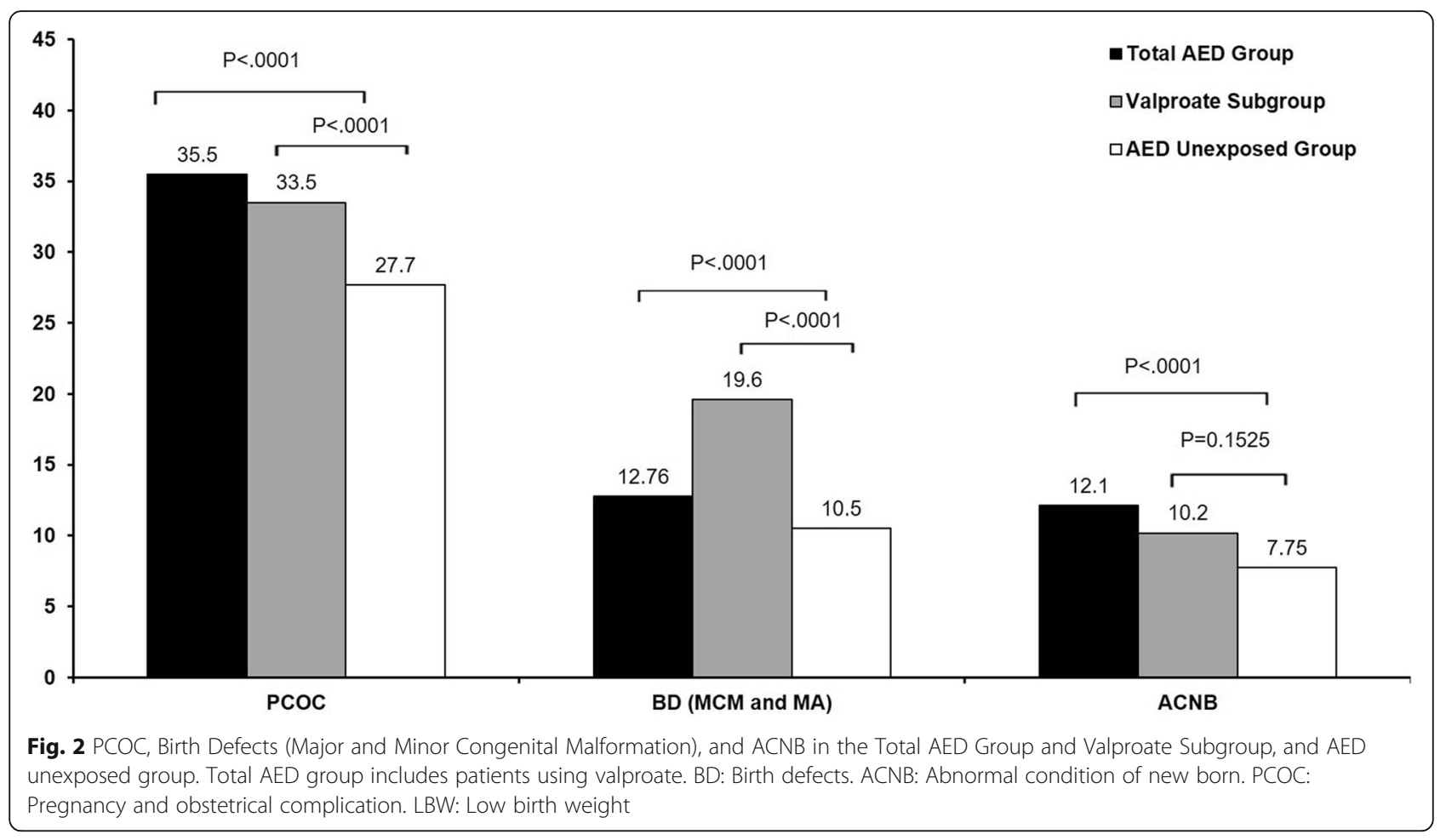

\section{Discussion}

The total AED group was significantly different from the AED unexposed group on all observed outcomes, whereas valproate subgroup differed statistically from AED unexposed group only on BD and PCOC. These two exposure groups had varied patterns of observed outcomes that were combined using the latent trait model. The psychometric properties of the combined outcome were evaluated and compared among the two exposed groups and one healthy comparison group. The

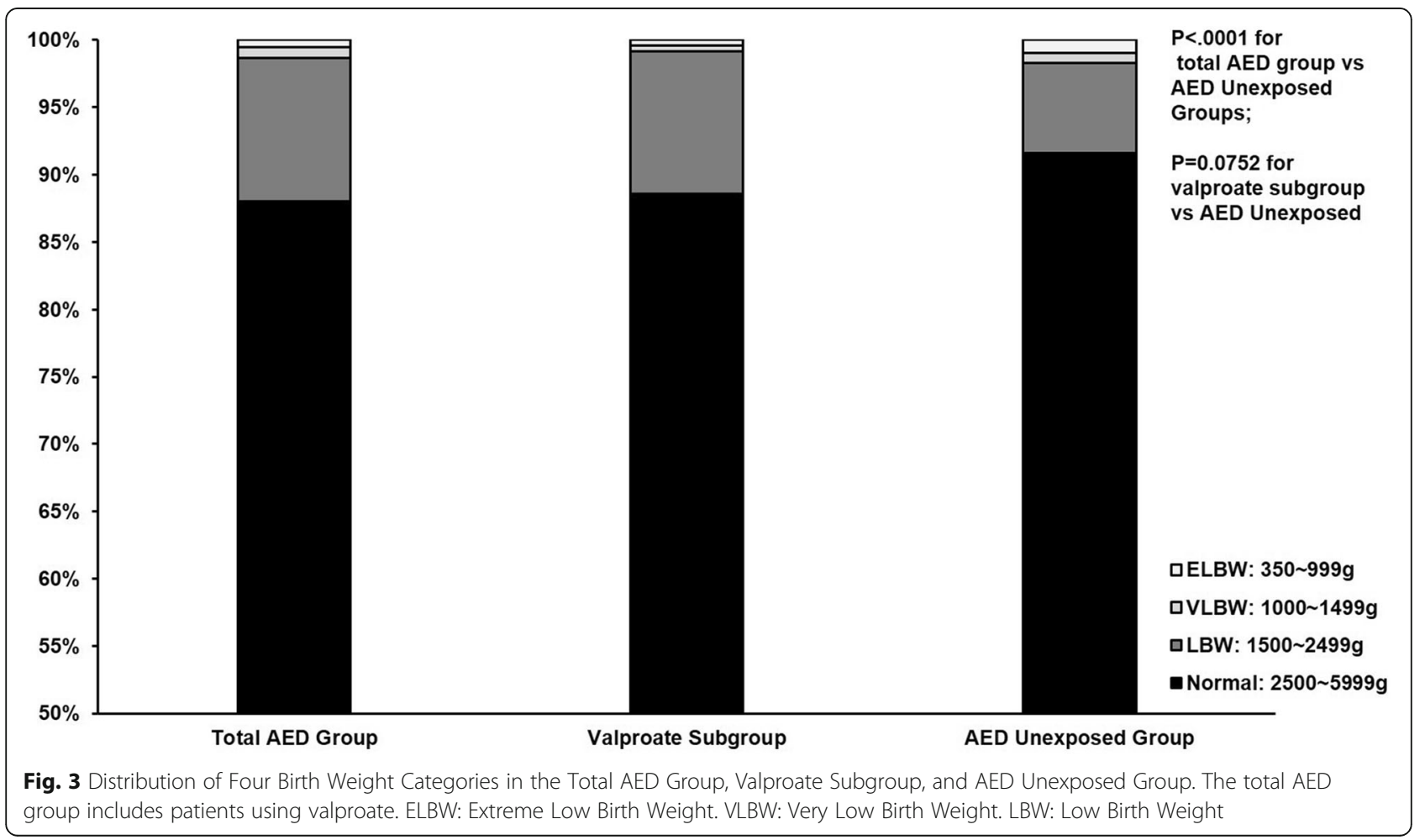


Table 2 Propensity Score Adjusted Drug Effects, and Importance of Each Component or Combined Outcome in Pregnant Women Exposed to Total AED or Valproate Only

\begin{tabular}{|c|c|c|c|}
\hline & Component Outcomes & $\begin{array}{l}\text { Propensity Score Adjusted Drug Effects } \\
(\beta \pm S E, P \text { Value) }\end{array}$ & $\begin{array}{l}\text { Spearman Correlation with the Length } \\
\text { of Hospital Stay during Delivery }(95 \% \mathrm{Cl}, P \text { Value }\end{array}$ \\
\hline \multirow[t]{5}{*}{ Total AED Group } & $\mathrm{BD}$ & $0.34 \pm 0.16, P=0.0356$ & $0.08(0.05 \sim 0.12, P<.0001)$ \\
\hline & ACNB & $0.60 \pm 0.15, P=0.0001$ & $0.08(0.04 \sim 0.11, P<.0001)$ \\
\hline & PCOC & $0.70 \pm 0.11, P<.0001$ & $0.24(0.20 \sim 0.27, P<.0001)$ \\
\hline & LBW & $0.10 \pm 0.02, P<.0001$ & $0.13(0.09 \sim 0.16, P<.0001)$ \\
\hline & $\mathrm{APO}$ & $0.24 \pm 0.03, P<.0001$ & $0.24(0.20 \sim 0.27, P<.0001)$ \\
\hline \multirow[t]{5}{*}{ Valproate Subgroup } & $\mathrm{BD}$ & $0.96 \pm 0.41, P=0.0196$ & $0.17(0.05 \sim 0.29, P=0.0066)$ \\
\hline & ACNB & $0.67 \pm 0.43, P=0.1223$ & $0.02(-0.10 \sim 0.15, P=0.7135)$ \\
\hline & PCOC & $0.99 \pm 0.32, P=0.0019$ & $0.16(0.03 \sim 0.28, P=0.0122)$ \\
\hline & LBW & $0.10 \pm 0.06, P=0.0856$ & $0.04(-0.09 \sim 0.16, P=0.5640)$ \\
\hline & $\mathrm{APO}$ & $0.32 \pm 0.09, P=0.0004$ & $0.16(0.03 \sim 0.28, P=0.0121)$ \\
\hline
\end{tabular}

Covariates include: mother's epilepsy diagnosis, mother's anxiety diagnosis, mother's bipolar diagnosis, mother's mental disorder diagnoses, mother's mental disorder diagnoses, mother's infection and parasitic diagnosis, mother's age, father's age, mother's education level, father's education level, mother's total number of prenatal visits, mother's parity, mother's marital status, mother's previous gestational diabetes, mother's previous gestational hypertension, mother's previous cesarean $B D$ Birth defects, ACNB Abnormal condition of new born, PCOC Pregnancy and obstetrical complication, $L B W$ Low birth weight, APO Adverse Perinatal and Pregnancy Outcome

four component outcomes were found to be not significantly different on the incidence rates. One exception was PCOC, which had the highest incidence out of the three component outcomes. However, neither the differences of AED effects on PCOC nor the correlation between PCOC and the length of the hospital stay during delivery was significantly different between the total AED group and AED unexposed group. Thus, these four component outcomes exhibited a high level of homogeneity and demonstrated the validity of component selection for the AED safety study.

Table 1 provides evidence for a pronounced difference between the mother-infant pairs exposed to AEDs versus AED unexposed group and raises a concern that studies of pregnancy outcome need to control for these differences. In our study, propensity score was used to adjust these covariates for drug effect assessment.

Figures 1, 2, and 3 raise concern for combining outcomes in valproate drug safety studies. Compared to the AED unexposed group, AED use in the total exposed group was associated with significant effects on all four component outcomes, whereas, valproate use was related to increased BD and PCOC, and had no significant effect on ACNB and BW. The lack of differences for valproate on APO, ACNB and BW may be in part due to the small sample size of the valproate subgroup. Previous studies using birth registry data has shown that fetal valproate exposure is associated with higher rates of $\mathrm{BD}$ than other AEDs. For example, the UK Birth Registry reported a $6.7 \%$ rate of major congenital malformations for valproate, and the North American AED Pregnancy Registry reported a rate of $10.7 \%[3,45]$. However, the incidence of minor abnormalities in our study, 9.1\% in AED unexposed group and $10.7 \%$ in the total AED group, was lower than reported in the literature, $15 \%$ to $20 \%$ in the general population and 37\% in AED exposed pregnant women [46-50]. Considering the difficulties of identifying minor abnormalities, under-reporting or misdiagnosis of minor abnormalities in claims data might account for this discrepancy. Given that valproate exposure is not consistently associated with the four component outcomes and violates the criteria for component selection for a composite outcome, a concern is raised about the validity of combining these four outcomes in a valproate safety study.

To our knowledge, combining outcomes using a latent variable model has not been utilized in any pharmacoepidemiological studies previously. This model was first described in 2008 for combining four birth defect outcomes to construct an infant morbidity index [11]. We employed the model to assess the comprehensive effects of AEDs on four adverse perinatal and pregnancy outcomes in both mothers and infants. Superior to other composite outcomes, the latent variable model generates a continuous measure that correlates to the component outcomes with different levels and takes into account the comprehensive effects of all component outcomes [11].

The final estimate of the latent variable $S^{\wedge}$ ranged from 0.08 for normal infant-mother pairs to 0.61 for the mother-infant pairs with different patterns of $\mathrm{BD}$, ACNB, PCOC, and ELBW. These estimates are similar in magnitude to those documented previously [11].

This article is based on a thesis published by one of the authors in 2013 (http://ufdc.ufl.edu/UFE0046207/00001).

\section{Study limitations}

Several limitations should be considered as a consequence of using linked claims data and the nature of the study. 
First, by combining MCM, MA, LBW, and PCOC, the latent variable APO is an overall adverse outcome for both mothers and infants. The association between drug exposure and each individual component outcome is unknown if latent variable APO is used as a dependent variable in the model. Second, the power to detect differences in the valproate only subgroup is a concern due to small sample size. The insignificant difference in APO between valproate only subgroup and health unexposed group might be due to the inadequate statistical power. Third, MAs might be underestimated in this study, which could cause underestimation of APO score. However, the misclassification of MAs is nondifferential, so it should not affect the assessment of differences between drug use groups. Finally, this latent variable model combines manifest outcomes based upon the probability of occurrence in the study population. The severity of each outcome is not mathematical weighted in the combining process. Future studies are needed to develop more advanced statistical models to combine more specific outcomes based upon not only the probability of occurrence, but also the severity of each outcome.

\section{Conclusions}

This study used a latent trait model to assess adverse pregnancy and perinatal outcomes in women exposed to antiepileptic drugs during pregnancy. We recommend using this latent trait model in other drug studies examining similarly related component outcomes. If the study drug, is only weakly associated with any of the selected component outcomes, the study drug's effects on the combined outcome may be diluted and be statistically non-significant compared to the reference group. Such an approach is detrimental to any drug safety study as the results move towards the null and the true teratogenic effects of the drug can be masked. Hence, evaluation of selected components is essential before a latent trait model can be used to assess a combined outcome.

\section{Additional file}

Additional file 1: Table S1. Operational Definition 625 for Component Outcomes. Table S2. Demographic Characteristics and Missing Data of Study Participants. Table S3. Observed Frequency (OBFREQ), Expected Frequency (EXFREQ), Observed Percents (OB\%), Expected Percents (EX\%), Estimates of Posterior Mean of the Latent Variable $S^{\wedge} 632$ and the APO by Combinations of Four Observed Outcomes. (DOCX $23 \mathrm{~kb}$ )

\footnotetext{
Abbreviations

ACNB: Abnormal Condition of Newborn; AED: Antiepileptic Drugs (also called Anticonvulsant Drugs); APO: Adverse Perinatal Outcome; BD: Birth defect; BW: Birth weight; Cl: Confidence Interval; ELBW: Extremely Low Birth Weight; LBW: Low birth weight; MA: Minor anomaly; MCM: Major congenital malformation; NBW: Normal Birth Weight; PCOC: Pregnancy and Obstetric Complication; SE: Standard Error; VLBW: Very Low Birth Weight
}

\section{Funding}

No funding support for this study.

\section{Availability of data and materials}

All data in this study were provided by Florida Department of Health (http://www.floridahealth.gov/) and the Agency for Health Care Administration (http://ahca.myflorida.com/).

\section{Authors' contributions}

XW Initiated and designed the study; made substantial contributions to conception, and acquisition, analysis and interpretation of data; drafted and revised the manuscript; and gave final approval of the version to be published. AH Made substantial contributions to conception and design, and acquisition of data; involved in drafting the manuscript and revising it critically for important intellectual content; and gave final approval of the version to be published. JAD Made substantial contributions to conception and design, analysis and interpretation of data; involved in revising the manuscript critically for important intellectual content; gave final approval of the version to be published. BB Made substantial contributions to conception and design, analysis and interpretation of data; involved in revising the manuscript critically for important intellectual content; gave final approval of the version to be published. XL Made substantial contributions to conception and design, analysis and interpretation of data; involved in revising the manuscript critically for important intellectual content; gave final approval of the version to be published. RE Made substantial contributions to conception and design, and interpretation of data; involved in revising the manuscript critically for important intellectual content; gave final approval of the version to be published. JR Made substantial contributions to conception and design, and acquisition of data; involved in revising the manuscript critically for important intellectual content; gave final approval of the version to be published. RS Made substantial contributions to conception and design, and interpretation of data; involved in revising the manuscript critically for important intellectual content; gave final approval of the version to be published. KJM Made substantial contributions to conception and design, and analysis and interpretation of data; involved in drafting the manuscript and revising it critically for important intellectual content; gave final approval of the version to be published.

\section{Competing interests}

The authors declare that they have no competing interests, except Dr. Kimford Meador. The followings are Dr. Meador's financial disclosure statements:

\section{Research Grants}

NIH/NINDS 2U01-NS038455-11A1. Meador (Multi-PI) "Maternal Outcomes and Neurodevelopmental Effects of Antiepileptic Drugs;"** $\mathrm{NIH} / \mathrm{NINDS}$ R01NS088748-01. Drane (PI) "Dissecting the Cognitive Roles of Hippocampus and Other Temporal Lobe Structures" Role: Co-l; NIH 1 R01 NS076665-01A1 Susan Marino (PI) "Characterizing and Predicting Drug Effects on Cognition" Role: Consultant;

Sunovion Pharmaceuticals. Meador (PI). "Double-Blind, Randomized, Two Period Crossover Comparison of the Cognitive and Behavioral Effects of Eslicarbazepine Acetate and Carbamazepine in Healthy Adults." Consultant

Consultant for the non-profit Epilepsy Study Consortium that receives monies from multiple pharmaceutical companies. Dr. Meador has consulted for the Epilepsy Study Consortium for the following companies: Eisai, GW Pharmaceuticals, NeuroPace, Novartis, Supernus, Upsher Smith Laboratories, UCB Pharma and Vivus Pharmaceuticals.

Other

He is Co-I and Director of Cognitive Core of the Human Epilepsy Project for the Consortium.

Note that the Epilepsy Study Consortium pays Dr. Meador's university for his consultant time.

Dr. Meador receives income from clinical EEG procedures and care of neurological patients.*

Note that those items with asterisk involve personal income in excess of $\$ 10,000$. 


\section{Ethics approval and consent to participate}

This study was approved by the Institutional Review Board of the University of Florida and the Florida Department of Health. To obtain the research data, we completed the data utilization agreements with Florida Department of Health, and Florida Agency for Health Care Administration.

\section{Author details}

'Health Outcomes, College of Pharmacy, University of Rhode Island, 7 Greenhouse Rd., Kingston, RI 02881, USA. ${ }^{2}$ Department of Pharmaceutical Outcome and Policy, University of Florida, Gainesville, FL, USA. ${ }^{3}$ Department of Epidemiology, University of Washington, Seattle, WA, USA. ${ }^{4}$ Department of Biostatistics, University of Florida, Gainesville, FL, USA. ${ }^{5}$ Department of Biostatistics \& Epidemiology, Systems, Population and Leadership, University of Michigan, Ann Arbor, Ml 48109, USA. ${ }^{6}$ Department of Obstetrics \& Gynecology, University of Florida, Gainesville, FL, USA. ${ }^{7}$ Department of Pediatrics, University of Florida, Gainesville, FL, USA. ${ }^{8}$ Department of Neurology \& Neurological Sciences, Stanford University, Stanford, CA, USA.

\section{Received: 10 July 2015 Accepted: 9 December 2016}

\section{Published online: 06 January 2017}

\section{References}

1. The Centers for Disease Control and Prevention. Birth Defects. http://www prevention-news.com/general/cdc-prevention.htm. Accessed 14 Jan 2011.

2. Meador KJ, Baker GA, Finnell RH, et al. In utero antiepileptic drug exposure: fetal death and malformations. Neurology. 2006;67:407-12.

3. Wyszynski DF, Nambisan M, Surve T, Alsdorf RM, Smith CR, Holmes LB. Increased rate of major malformations in offspring exposed to valproate during pregnancy. Neurology. 2005;64:961-5.

4. Mølgaard-Nielsen D, Hviid A. Newer-generation antiepileptic drugs and the risk of major birth defects. JAMA. 2011;305(19):1996-2002.

5. Bakker MK, Kerstjens-Frederikse WS, Buys CH, de Walle HE, de Jong-van den Berg LT. First-trimester use of paroxetine and congenital heart defects: a population-based case-control study. Birth Defects Res A Clin Mol Teratol. 2010;88(2):94-100.

6. Davis RL, Rubanowice D, McPhillips H, Raebel MA, Andrade SE, Smith D, Platt R. HMO Research Network Center for Education, Research in Therapeutics. Risks of congenital malformations and perinatal events among infants exposed to antidepressant medications during pregnancy. Pharmacoepidemiol Drug Saf. 2007;16(10):1086-94.

7. McCulloch C. Joint modelling of mixed outcome types using latent variables. Stat Methods Med Res. 2008;17:53-73.

8. Rabe-Hesketh S, Skrondal A. Classical latent variable models for medical research. Stat Methods Med Res. 2008:17:5-32

9. Rabe-Hesketh S, Skrondal A. Multilevel and Latent Variable Modeling with Composite Links and Exploded Likelihoods. Psychometrika. 2007;72(2):123-40.

10. Sobel ME. Measurement, Causation and local independence in latent variable models. In: Latent Variable Modeling and Applications to Causality, Lecture Notes in Statistics, Volume 120. 1997. p. 11-28.

11. Liu X, Roth J. Development and validation of an infant morbidity index using latent variable models. Stat Med. 2008;27(7):971-89.

12. Cordoba G, Schwartz L, Woloshin S, Bae H, Gotzsche C. Definition, reporting, and interpretation of composite outcomes in clinical trials: systematic review. BMJ. 2010;341:c3920.

13. Freemantle N, Calvert M, Wood J, Eastaugh J, Griffin C. Composite outcomes in randomized trials: greater precision but with greater uncertainty? JAMA. 2003;289(19):2554-9.

14. O'Leary-Kelly SW, Volurka RJ. The empirical assessment of construct validity. J Oper Manag. 1998;16:387-405.

15. Persaud TVN, Chudley AE, Skalko RG. Basic concepts in teratology. New York: Alan R. Liss, Inc.; 1985

16. Cooper WO, Hernandez-Diaz S, Arbogast PG, Dudley JA, Dyer S, Gideon PS, Hall K, Ray WA. Major congenital malformations after first-trimester exposure to ACE inhibitors. N Engl J Med. 2006;354(23):2443-51.

17. Costei AM, Kozer E, Ho T, Ito S, Koren G. Perinatal outcome following third trimester exposure to paroxetine. Arch Pediatr Adolesc Med. 2002;156(11): 1129-32.

18. Cooper WO, Hernandez-Diaz S, Gideon P, Dyer SM, Hall K, Dudley J, Cevasco $M$, Thompson AB, Ray WA. Positive predictive value of computerized records for major congenital malformations. Pharmacoepidemiol Drug Saf. 2008;17:455-60.
19. Salemi JL, Tanner JP, Block S, Bailey M, Correia JA, Watkins SM, Kirby RS. The relative contribution of data sources to a birth defects registry utilizing passive multisource ascertainment methods: does a smaller birth defects case ascertainment net lead to overall or disproportionate loss? J Registry Manag. 2011;38(1):30-8.

20. Wang Y, Sharpe-Stimac M, Cross PK, Druschel CM, Hwang SA. Improving case ascertainment of a population-based birth defects registry in New York State using hospital discharge data. Birth Defects Res A Clin Mol Teratol. 2005;73(10):663-8.

21. Reichman NE, Hade EM. Validation of birth certificate data. A study of women in New Jersey's HealthStart program. Ann Epidemiol. 2001;11(3):186-93.

22. Roohan PJ, Josberger RE, Acar J, Dabir P, Feder HM, Gagliano PJ. Validation of birth certificate data in New York State. J Community Health. 2003;28(5): $335-46$

23. Gore DC, Chez RA, Remmel RJ, Harahan M, Mock M, Yelverton R. Unreliable medical information on birth certificates. J Reprod Med. 2002;47(4):297-302.

24. DiGiuseppe DL, Aron DC, Ranbom L, Harper DL, Rosenthal GE. Reliability of birth certificate data: a multi-hospital comparison to medical records information. Matern Child Health J. 2002;6(3):169-79.

25. Northam S, Knapp TR. The reliability and validity of birth certificates. J Obstet Gynecol Neonatal Nurs. 2006;35(1):3-12.

26. Horn SD, Sharkey PD, Buckle JM, et al. The relationship between severity of illness and hospital length of stay and mortality. Med Care. 1991;29:305-17.

27. Hogan DP, Park JM. Family factors and social support in the developmental outcomes of very low-birth weight children. Clin Perinatol. 2000;27:433-59.

28. Duong HT, Hoyt AT, Carmichael SL, Gilboa SM, Canfield MA, Case A, McNeese ML, Waller DK, and the National Birth Defects Prevention Study. Is maternal parity an independent risk factor for birth defects? Birth Defects Res A Clin Mol Teratol. 2012;94(4):230-6.

29. Baardman ME, Kerstjens-Frederikse WS, Corpeleijn E, de Walle HE, Hofstra RM, Berger RM, Bakker MK. Combined adverse effects of maternal smoking and high body mass index on heart development in offspring: evidence for interaction? Heart. 2012;98(6):474-9. Epub 2012 Jan 30.

30. Siega-Riz AM, Herring AH, Olshan AF, Smith J. Moore C; National Birth Defects Prevention Study. The joint effects of maternal prepregnancy body mass index and age on the risk of gastroschisis. Paediatr Perinat Epidemiol. 2009;23(1):51-7.

31. Andrade SE, Raebel MA. Use of prescription medications with a potential for fetal harm among pregnant women. Pharmacoepidemiol Drug Saf. 2006; 15(8);546-54

32. Koren G, Pastuszak A, Ito S. Drugs in Pregnancy. N Engl J Med. 1998;338: 1128-37.

33. Bracken MB, Holford TR. Exposure to prescribed drugs in pregnancy and association with congenital malformations. Obstet Gynecol. 1981;58(3):336-44.

34. Davis RL, Rubanowice D, McPhillips H. HMO Research Network Center for Education, Research in Therapeutics. Risk of congenital malformations and perinatal events among infants exposed to antidepressant medications during pregnancy. Pharmacoepidemiol Drug Saf. 2007;16:1086-94.

35. Niebyl JR, Simpson JL. Teratology and Drugs in Pregnancy, Gynecology and Obstetrics. Volume 2, Chapter 6. http://www.glowm.com/resources/glowm/ cd/pages/v2/v2c006.html. Accessed 26 Dec 2012.

36. Chung W. Teratogens and their effects. https://www.cdc.gov/niosh/docs/99104/pdfs/99-104.pdf. Accessed 26 Dec 2012

37. The Effects of Workplace Hazards on Female Reproductive Health. DHHS (NIOSH) Publication No. 99.104. Feb 1999. https://www.cdc.gov/niosh/docs/ 99-104/pdfs/99-104.pdf. Accessed 26 Dec 2012.

38. Maternal Disease and Complications-Summary Outline. https://www.yumpu. com/en/document/view/21948441/maternal-disease-and-complicationssummary-outline. Accessed 26 Dec 2012.

39. Bartholomew DJ, Knott M. Latent variable models and factor analysis. London: Arnold; 1999.

40. Hartley HO. The modified Gauss-Newton method for the fitting of nonlinear regression functions by least squares. Techmetrics. 1961;3:269-80.

41. Yen WM. Scaling performance assessments: Strategies for managing local item dependence. J Educ Meas. 1993;30:187-213.

42. Kimberlin C, Winterstein AG. Validity and reliability of measurement instruments use in research. Am J Health-Syst Pharm. 2008;65:2276-84.

43. John OP, Benet-Martinez V. Measurement: Reliability, construct validation, and scale construction. In: Reis HT, Judd CM, editors. Handbook of research methods in social psychology. New York: Cambridge University Press; 2000. p. 339-69. 
44. Wen X, Meador KJ, Hartzema A. Antiepileptic drug use by pregnant women enrolled in Florida Medicaid. Neurology. 2015;84(9):944-50. Epub 2015 Feb 4.

45. Campbell E, Kennedy F, Russell A, Smithson WH, Parsons L, Morrison PJ, Liggan B, Irwin B, Delanty N, Hunt SJ, Craig J, Morrow J. Malformation risks of antiepileptic drug monotherapies in pregnancy: updated results from the UK and Ireland Epilepsy and Pregnancy Registers. J Neurol Neurosurg Psychiatry. 2014;85(9):1029-34. doi:10.1136/jnnp-2013-306318.

46. Kaneko S, Kondo T. Antiepileptic agents and birth defects: incidence, mechanisms and prevention. CNS Drugs. 1995;3(1):41-55.

47. Women with epilepsy: drug risks and safety during pregnancy. American academy of neurology. 2009. https://www.aan.com/Guidelines/Home/ GetGuidelineContent/338, Accessed 2 Jan 2011.

48. Samren EB, van Duijn CM, Christianens GC, et al. Antiepileptic drug regimens and major congenital abnormalities in the offspring. Ann Neurol. 1999:46:739-46.

49. Kaneko S, Battino D, Andermann E, et al. Congenital malformations due to antiepileptic drugs. Epilepsy Res. 1999;33:145-58.

50. Kaneko S, Otani K, Fukushima Y, et al. Teratogenicity of antiepileptic drugs: analysis of possible risk factors. Epilpesia. 1988;29:459-67.

Submit your next manuscript to BioMed Central and we will help you at every step:

- We accept pre-submission inquiries

- Our selector tool helps you to find the most relevant journal

- We provide round the clock customer support

- Convenient online submission

- Thorough peer review

- Inclusion in PubMed and all major indexing services

- Maximum visibility for your research

Submit your manuscript at www.biomedcentral.com/submit
Biomed Central 